\title{
DETECTION OF POWER GRID HARMONIC POLLUTION SOURCES BASED ON UPGRADED POWER METERS
}

\author{
Predrag Petković ${ }^{*}$ - Dejan Stevanović ${ }^{* *}$
}

\begin{abstract}
The paper suggests a new and efficient method for location of nonlinear loads on a grid. It is based on measuring of distortion power. The paper reviews different definitions of distortion power and proves that the method is feasible independently on particular definition. The obtained results of simulation and measurement confirm the effectiveness and applicability of the method. The proposed solution is suitable for software update of existing electronic power-meters or can be implement as hardware upgrade.
\end{abstract}

K e y w or d s: distortion power, harmonic pollution sources detection, electronic power meter

\section{INTRODUCTION}

The end of the previous century improved our lives with innovative smart electronic appliances. Simultaneously, the electronic control systems became unavoidable units in industrial equipment that provide low cost mass production. Most of electronic devices and apparatus operate with DC supply. In contrary, the common power grid provides $\mathrm{AC}$ voltage. Consequently, every electronic equipment connected to the mains requires $\mathrm{AC}$ to $\mathrm{DC}$ converter. Today they are the most common loads at power grid. However their nonlinear nature produces harmonics that enter into the grid causing numerous unwanted harms [1], [2], [3]. The long-lasting growth of nonlinear loads intensified the problems caused by harmonics. In order to suppress their devastating effects to the grid, every country introduced own standard that limits the acceptable amount of each harmonic. Two the best known and widely applied standards in this area are the IEEE 519-1992 and IEC 61000 series [1], [3]. The standard IEC/EN61000-3-2 entered into force in the European Union. It states the boundaries for the permissible nonlinear distortion of the current up to the 40-th harmonic. The standard is related to the distortion produced by electronic and electrical appliances in households. It covers loads up to $16 \mathrm{~A}$ per phase supplied with voltage up to $415 \mathrm{~V}$. The standards normalize restrictions for the harmonics pollution but do not identify what happens if a customer exceeds them. Practically, there are two possible cases: the first is to disconnect such customer. However, that is traumatic for customer and not profitable for utility. The other solution is to charge the harmonics producers a penalty tax if they exceed allowable limits. The extra fee should be related to the level of pollution. The tax driven instruction may be blocked by two technical problems: the detection of the amount and location of harmonic pollution, and isolation of the system from the effects of impedance variation. Seriousness of the problem provoked many experts to find effective solution. The core issue that prevents finding the overall solution to this problem lies in divergence within the power engineering community on the best definition of the harmonic pollution level [4]. Using THD factor (Total Harmonic Distortion) is not appropriate for evaluating the effects of the pollution load at power grid [4]. Namely, THD only defines how much the waveform of current/voltage is distorted. It does not give evidence of nominal power of the load. Drawbacks of THD provoked finding other solutions to define Power Quality Index (PQI). Detecting the source of harmonic pollution becomes very urgent and should be solved as soon as possible. The authors of this paper are motivated to offer a relatively simple solution that could be implemented as an upgrade to household power meters. Guided by this aim we propose a new method capable to locate source of harmonic pollution on the grid at users connection point. Simultaneously it provides the utility information about the amount of the pollution entered from each specific customer.

\section{BASIC DEFINITION OF ELECTRIC POWER QUANTITIES}

\subsection{Sinusoidal condition}

The definitions of the active, reactive, and apparent power in pure sinusoidal conditions are widely known.

$$
\begin{gathered}
P=V_{\mathrm{RMS}} I_{\mathrm{RMS}} \cos (\theta), \\
Q=V_{\mathrm{RMS}} I_{\mathrm{RMS}} \sin (\theta)
\end{gathered}
$$

where $V_{\mathrm{RMS}}$, and $I_{\mathrm{RMS}}$ are RMS values of the voltage and current, respectively; $\theta$ is the phase angle between the voltage and current. Vector sum of active and reactive power gives the phasor power $S$ [5]. Its magnitude is

$$
S=\sqrt{P^{2}+Q^{2}} .
$$

\footnotetext{
* University of Niš, Faculty of Electronic Engineering, Aleksandra Medvedeva 14, 18000 Ni, Serbia, predrag.petkovic@elfak.ni.ac.rs, ** Innovation Centre of Advanced Technologies CNT lmt., Serbia, dejan.stevanovic@icnt.rs
} 
The magnitude of phasor power was known as apparent power for many years. However, some novel definitions recognize the phasor power $S$, as the apparent power only for sinusoidal voltages and currents. Otherwise the apparent power differs from $S$ and is denoted as $U$, [5].

\subsection{Nonsinusoidal condition}

In the presence of nonlinear loads, however, the system no longer operates in sinusoidal condition. The effect of harmonics should be taken into account. When harmonics exist in the power supply system, the instantaneous values of the voltage and current can be express as

$$
x(t)=\sum_{h=1}^{M} X_{h} \sin \left(\omega_{h} t+\alpha_{h}\right),
$$

where $h$ is the number of harmonic, $M$ denotes the highest harmonic, while $X_{h}, \omega_{h}$ and $\alpha_{h}$ are the amplitude of the signal (voltage or current), frequency and phase angles of the $h$-th harmonic, respectively. RMS values for signal expressed by (4), is given as

$$
X_{\mathrm{RMS}}=\sqrt{\sum_{h=1}^{M} X_{\mathrm{RMS}_{h}}^{2}}
$$

where $X_{\mathrm{RMS}_{h}}$ is the RMS values of the $h$-th harmonic. Product of the voltage and current on the same harmonic frequency gives the harmonic power. Total active power is defined as

$$
P=\sum_{h=1}^{M} V_{\mathrm{RMS}_{h}} I_{\mathrm{RMS}_{h}} \cos \left(\theta_{h}\right)
$$

Total active power could be presented as a sum of components related to fundamental and other harmonics

$$
P=P_{1}+P_{\mathrm{H}}
$$

where $P_{1}$ is known as the fundamental active power because it is related to fundamental harmonic $(h=1) ; P_{H}$ comprises sum of all harmonic components for $(h \geq 2)$ and is referred to as harmonic active power.

According to Budeanu $[3,5]$ reactive power is defined as

$$
Q_{\mathrm{B}}=\sum_{h=1}^{M} V_{\mathrm{RMS}_{h}} I_{\mathrm{RMS}_{h}} \sin \left(\theta_{h}\right)=Q_{1}+Q_{\mathrm{H}}
$$

where, similarly to (7), $Q_{1}$ stands for fundamental active power and $Q_{\mathrm{H}}$ denotes harmonic reactive power. Many authors argued about the usefulness of $Q_{\mathrm{B}}$ for quantifying the flow of harmonic nonactive power [6], [7]. However, according to [8], the "postulates of Czarnecki have not won universal recognition". Field measurements and simulations proved that in many situations $Q_{\mathrm{H}}<0$, leading to cases where $Q_{\mathrm{B}}<Q_{1}$, [7]. Even if negative, the reactive power contributes to the losses in the same way as the positive reactive powers. As harmonic reactive powers of different orders appear at diverse frequencies it is easy to conclude that the reactive powers should not be added arithmetically (as recommended by Budeanu) [7].
Thereafter Standard IEEE Std 1459-2010 suggests reactive power to be calculated as

$Q_{\mathrm{IEEE}}=\sqrt{\sum_{h=1}^{M}\left[V_{\mathrm{RMS}_{h}} I_{\mathrm{RMS}_{h}} \sin \left(\theta_{h}\right)\right]^{2}}=\sqrt{Q_{1}^{2}+\sum_{h=2}^{M} Q_{h}^{2}}$.

Equation (9) solves the problem when the value of the total reactive power $Q_{\mathrm{B}}$ is less than the value of the fundamental component $Q_{1}$. Moreover, additional definitions for reactive power where suggested, as well [9]: Fryze's reactive power

$$
Q_{\mathrm{F}}=\sqrt{U^{2}-P^{2}}
$$

and Sharon's reactive power

$$
Q_{\mathrm{S} h}=V_{\mathrm{RMS}} \sqrt{\sum_{h=1}^{M} I_{\mathrm{RMS}_{h}}^{2} \sin ^{2}\left(\theta_{h}\right)} .
$$

Nevertheless, the expression (8) is used widely and is a part of IEEE Standard Dictionary 100-1996. Therefore it is practically implemented in most of instruments for reactive power metering. Consequently we accepted (8) as a reference. It is important to note that in reality, harmonics contribute to active and reactive power with less than $3 \%$ of the total active or reactive power [5]. Oppositely, they considerably contribute the total power balance, as will be presented in this paper.

The harmonic component has main impact on the distortion power $D$. This term has been introduced by $\mathrm{C}$. Budeanu in 1927, [10]. The phasor power is vector sum of active and reactive power as indicates (3). However, this is true only for sinusoidal conditions. In presence of harmonics it should be applied to each harmonic component of active and reactive power separately. In addition, the apparent power $U$ represents a vector sum of phasor power and distortion power [5]. Accordingly, its intensity is calculated as

$$
U=\sqrt{S^{2}+D^{2}}=I_{\mathrm{RMS}} V_{\mathrm{RMS}} .
$$

\section{REVIEW OF METHODS FOR LOCATING SOURCES OF HARMONIC POLLUTION}

Contemporary power grid community recognizes several methods capable to detect sources of harmonics. In general they could be distinguished as: (i) multi-point methods, and (ii) single-point methods.

The first uses distributed and synchronous measurement system and collect data from different points at power grid. It provides accurate and comprehensive information about harmonic propagation at the grid. Unfortunately its practical realization is difficult due to requirements for complex and expensive measurement instrumentation. In contrary, single-point methods are more suitable for application, but less exact. The most common single-point strategy is to track the sign of $P_{\mathrm{H}}[1],[10]$, [11]. If it is negative $\left(P_{\mathrm{H}}<0\right)$, generator of harmonics is on the consumer's side. In opposite case, if $P_{\mathrm{H}}>0$, then the utility-side caused harmonic distortion. This method is widespread and has been used for many years [1], [11]. A number of manufactures of metering equipment exposes this possibility as a key feature of their equipment [11]. 


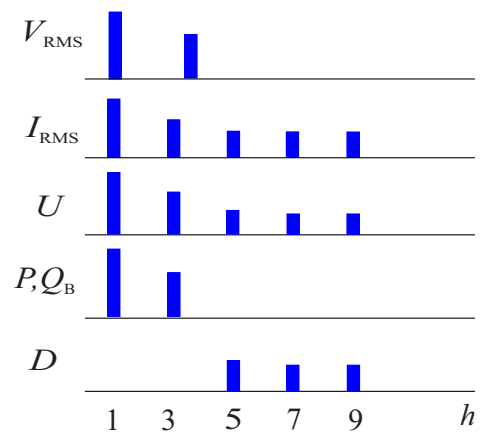

Fig. 1. Spectral components of voltage, current and power on a nonlinear load

However, further research showed that the method is not $100 \%$ accurate $[11]$.

Therefor the method was upgraded to track the sign of the harmonic reactive power, $Q_{\mathrm{H}}$. This method is a complement to the previous. Which of them $\left(P_{\mathrm{H}}\right.$ or $\left.Q_{\mathrm{H}}\right)$ will be applied depends on the balance of resistances and reactance of the loads [1], [11]. Unfortunately, determining the type of impedance is, usually a very difficult problem.

An alternative group of methods capable to locate the source of harmonics relies on measurement of impedance of the grid and customer harmonic impedances. Theoretically, the method is reliable. Therefore it appears in many variations [12]. However this method cannot be implemented easily. The practical problem is that one can determine the impedances only if intrudes the system. Therefore the method is costly and not suitable for wider implementation.

A different approach is based on comparison of three non-active power components [13]. The method has been improved by the same authors in [14].

The authors of recently published paper claim that $T H D$ is not sufficient for reliable detection of dominant nonlinear loads. Therefore, they introduces new power quality index $(P Q I)$ to monitor the affect of each nonlinear load on a point of common coupling of a power distribution system [4]. It is defined as a product of Load Composition Rate $(L C R)$ and THD. Both parameters are derived from the load current waveform. They use own $R M P$ (reduced multivariate polynomial) model to estimate $L C R$ and THD. Product of these values are used for harmonic pollution ranking each particular loads.

\section{A NEW METHOD FOR LOCATING HARMONIC POLLUTION SOURCE BASED ON DISTORTION POWER METERING}

In this paper we suggest a very obvious but completely new solution appropriate to identify the location of cause responsible for harmonic pollution on the network. The method is based on the calculation of distortion power $D$ according to (12) as

$$
D=\sqrt{U^{2}-P^{2}-Q^{2}} .
$$

The method will be explained on an example. Let us observe a nonlinear load connected to a power system supplied with voltage distorted by a 3-rd harmonic as Fig. 1 shows in the top. Let assume that due to the nonlinearity, the current trough the load has harmonics $3,5,7,9$ as presented in second diagram from the top in Fig.1.

All harmonics will affect the apparent power $U$. As specified in Section 2, $P$ and $Q_{\mathrm{B}}$ have only harmonics produced at the same frequencies of voltage and current namely fundamental and the third harmonic. However, the distortion power contains all other harmonics. This is so because $D$ is caused by voltages and currents with different frequencies. The lower part of Fig. 1 illustrates the spectral components of $U, P, Q_{\mathrm{B}}$ and $D$, respectively.

It is obvious that $D>0$ unquestionably means that a nonlinear load is connected at the grid. This is the essence of our method. The most important benefit of the solution is that it is suitable to be implemented in electronic power meters. Practically, most of contemporary solid state meters are able to register $P, Q_{\mathrm{B}}$ and $U$. Consequently only a slight intervention within the software (or DSP hardware) enriches the meter with the possibility to provide $D$ according to (13). It is clear that the value of $Q$ in presence of harmonics depends on the applied definition (8), (9), (10) or (11).

According to the different definitions for $Q$ one can claim the accuracy of the approach. Therefore it is important to stress that the goal is to provide the utility with means to locate and discipline harmonics producers. In that course it is needed and sufficient to quantify the amount of the distortion. According to Standard IEC 62052-11, commercial electronic meters register $Q$ with accuracy less than for $P$. Accordingly, (13) will provide lower accuracy comparing to $P$.

The proposed method guaranties that any definition for $Q$, is good enough to give $D$ is proportional to the level of harmonic distortion. Any discrepancies among different values of $D$ (caused with definitions of $Q$ ) will produce systematic measurement errors. It is important to say that they will not discriminate any customer. To prove the statement it is sufficient to consider possible measurement errors. One is to omit a value of a quantity when it does exist. The second is to register some amount of $D$ even if it does not exist. According to the essence of the method the first error matches to the case when we register $D=0$ on a real nonlinear load. The second corresponds to case when a meter register $D>0$ in cases of linear loads.

We claim that, according to $(7), I_{\mathrm{RMS}}$ in presence of harmonics is always larger than $I_{\mathrm{RMS}}$ of the fundamental harmonic. Consequently the first error is not possible because $U^{2}$ will be always larger than $\left(P^{2}+Q^{2}\right)$ in presence of harmonics. The second is potentially possible and needs a deeper analysis that will be outlined in the next.

The key weakness of Budeanu's definition of $Q_{\mathrm{B}}$ in context of $D$ is that there are cases when $D>0$ even if the load is linear. This may occur only if the voltage is already distorted and the load has a reactive component. However Standard IEEE 519-1992 limits voltage THD on 
Table 1. Result of simulations

\begin{tabular}{lccccccc}
\hline & ILB & FL & ECFL & PCFL & 3-DR & SMPS & PWMVSD \\
\hline$I_{\text {RMS }}(\mathrm{A})$ & 0.44 & 0.1 & 0.09 & 0.13 & 13.53 & 14.84 & 14.23 \\
$V_{\mathrm{RMS}}(\mathrm{V})$ & 230.1 & 230.1 & 230.1 & 230.1 & 230.1 & 230.1 & 230.1 \\
$P_{1}(\mathrm{~W})$ & 100.05 & 17.31 & 18.59 & 16.09 & 2251.39 & 2249.74 & 2300 \\
$P_{\mathrm{H}}(\mathrm{W})$ & 0.09 & 0.01 & -0.06 & -0.14 & 0 & -39.52 & 3.11 \\
$P(\mathrm{~W})$ & 100.14 & 17.31 & 18.53 & 15.95 & 2251.39 & 2210.22 & 2303.11 \\
$Q_{1}(\mathrm{VAR})$ & 0 & 15.15 & -6.04 & -10.06 & 470.34 & 478.2 & 0 \\
$Q_{\mathrm{H}}(\mathrm{VAR})$ & 0 & 0.14 & 0.03 & 0.47 & 0 & -39.52 & -5.38 \\
$Q_{\mathrm{B}}(\mathrm{VAR})$ & 0 & 15.29 & -6.01 & -9.58 & 470.34 & 438.68 & -5.38 \\
$Q_{\text {IEEE }}(\mathrm{VAR})$ & 0 & 15.15 & 6.04 & 10.07 & 470.34 & 479.83 & 5.38 \\
$U(\mathrm{VA})$ & 100.14 & 23.6 & 20.9 & 29.69 & 3112.95 & 3414.14 & 3274.51 \\
$T H D_{\mathrm{V}}(\%)$ & 3 & 3 & 3 & 3 & 3 & 3 & 3 \\
$T H D_{\mathrm{I}}(\%)$ & 3 & 22.85 & 37.68 & 120.23 & 91.12 & 109.61 & 101.25 \\
$D_{\mathrm{B}}(\mathrm{VAR})$ & 0 & 4.86 & 7.57 & 23.13 & 2097.72 & 2564.92 & 2327.68 \\
$D_{\text {IEEE }}(\mathrm{VAR})$ & 0 & 5.28 & 7.54 & 22.93 & 2097.72 & 2557.55 & 2327.68 \\
$D_{1}(\mathrm{VAR})$ & 0 & 5.28 & 7.54 & 22.93 & 2097.72 & 2557.85 & 2327.68 \\
$D_{\mathrm{I}}(\mathrm{VAR})$ & 3 & 5.26 & 7.37 & 22.81 & 2095.65 & 2521.08 & 2328.69 \\
\hline
\end{tabular}

the grid to less than $5 \%$. As a consequence, the matching margin of $D$ is quite low. Doubtlessly the utility has interest to retain distortion as small as possible in order to save expensive equipment. Thus, the further analysis relies on the assumption that voltage $T H D<5 \%$.

In case of pure reactive and pure active loads $D=0$, according to (13). However if voltage is distorted, it is easy to prove mathematically that some $D>0$ will be registered. It is interesting to apprise of the errors caused by different definitions of $D$ using $Q_{\mathrm{B}}, Q_{\mathrm{IEEE}}$ and $Q_{1}$. For all cases, the minimum, almost zero error, was registered for pure active loads. The worst case arises for loads with phase angle of $\theta_{1}=(2 k+1) \pi / 2$. Even in that case relative errors in distortion power with respect to the phasor power $U$ were for $D_{\mathrm{B}} 2 \%$, for $D_{\text {IEEE }}$ and $D_{1} 3.16 \%$ of $U$.

Obviously Budeanu's definition of $Q$ produced minimal error. In practical applications, a meter can be calibrated not to register distortion power for values less than a threshold specified by utility $\left(D_{t}\right)$. Practically, the utility would specify a limit of acceptable $D$ that will not jeopardize the power system. This follows the regulative for tolerable harmonic amount in almost all contemporary electronic equipment. Therefore, this principle prevents the utility to charge the customer for using appliances with permitted volume of harmonics.

Budeanu's definition of $D$ is prone to some error if the signs of harmonic components of reactive power are opposite. Due to possible cancellation of harmonics with opposite sign, the total $Q_{\mathrm{B}}$ can be less than $Q_{1}$. Even then, as $U$ does not depend on $Q_{\mathrm{B}}$ the registered $D$ will increase when $Q_{\mathrm{B}}$. From the point of the utility this is sufficient to locate the harmonic producer.

Independently on used definition for reactive power $\left(Q_{\mathrm{B}}, Q_{\mathrm{IEEE}}\right.$ or $\left.Q_{1}\right)$, the method indubitably identify the location of the nonlinear source if $D>D_{t}$. Unlike all other methods, this approach does not involve spectral analysis of voltage and current [1], [10], [11]. All we need are values of $U, P$, and $Q_{\mathrm{B}}$ that are easily calculated from measured samples of voltage and current. All of them are available in almost all electronic power meters. Therefore only minor intervention is needed to adapt the meters to identify sources of harmonic pollution. Thereafter the utility will be capable to warn, punish with extra fee or even to disconnect the harmonic producers from the grid.

In order to prove the efficiency of proposed method the authors simulated behavior of the most frequently used benchmark loads. However the final verification was done by measurement on real loads using industrial power meter. The following section reviews simulation and measurement results.

\section{SIMULATION AND MEASUREMENT RESULTS}

\subsection{Simulation Results}

The authors were unable to resist the temptation to use MATLAB software in order to confirm the basic idea on the quickest and easiest way. Therefore equations (5), (6), (8), and (13) were coded as an original script. Aiming to simulate realistic cases, we assumed that the voltage contains 3rd harmonics whose amplitude is $3 \%$ of fundamental. This corresponds to the sine characterized with $50 \mathrm{~Hz}$ and $230 \mathrm{~V}$ RMS but with flattened top and bottom peaks of the sine-wave. We considered the following seven different types of loads connected to the grid:

a) Incandescent light bulb (ILB),

b) Fluorescent lamp (FL),

c) EcoBulb Compact Fluorescent Lamp (ECFL),

d) Phillips Compact Fluorescent Lamp (PCFL),

e) 6-pulse 3-phase diode rectifier dc power supply (3DR)),

f) 6-pulse switched-mode power supply (SMPS),

g) 6-pulse PWM controlled variable speed drive (PWM VSD). 


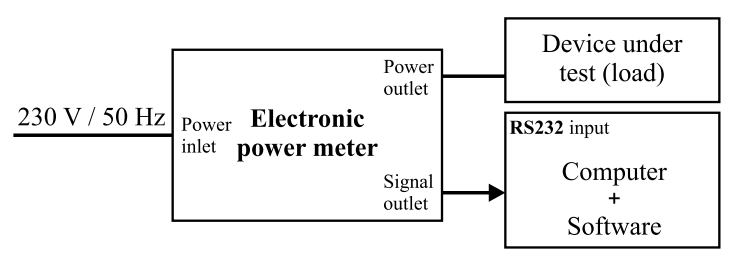

Fig. 2. Set-up circuit for distortion power measurement

Table 2. Measurement results for different types of energy saving lamps

\begin{tabular}{lcccccc}
\hline \multicolumn{1}{c}{ Load } & $\begin{array}{c}V_{\mathrm{RMS}} \\
(\mathrm{V})\end{array}$ & $\begin{array}{c}I_{\mathrm{RMS}} \\
(\mathrm{A})\end{array}$ & $\begin{array}{c}U \\
(\mathrm{VA})\end{array}$ & $\begin{array}{c}P \\
(\mathrm{~W})\end{array}$ & $\begin{array}{c}Q_{\mathrm{B}} \\
(\mathrm{VAR})\end{array}$ & $\begin{array}{c}D_{\mathrm{B}} \\
(\mathrm{VAR})\end{array}$ \\
\hline CFL20W bulb & 218.55 & 0.13 & 29.07 & 18.30 & -8.81 & 20.79 \\
CFL20W tube & 216.91 & 0.11 & 24.08 & 13.86 & -7.15 & 18.34 \\
CFL15W helix & 218.55 & 0.15 & 32.13 & 18.95 & -10.26 & 23.83 \\
CFL11W tube & 221.27 & 0.08 & 17.92 & 10.76 & 5.74 & 13.13 \\
CFL11WE14 & 215.51 & 0.08 & 17.24 & 10.79 & -5.26 & 12.38 \\
LED 10W Par lamp & 217.51 & 0.114 & 24.5 & 12.89 & -2.74 & 21.01 \\
LED Bulb 8W & 218.02 & 0.08 & 18.10 & 9.70 & -2.84 & 15.01 \\
LED Bulb 6W & 217.85 & 0.045 & 9.80 & 8.34 & -0.16 & 5.15 \\
LED Bulb 3W & 217.9 & 0.034 & 7.41 & 3.96 & -0.89 & 6.20 \\
CRT-21 HM903DT & 220.92 & 0.37 & 82.40 & 77.84 & -17.11 & 20.94 \\
Dell 1909W & 221.79 & 0.20 & 43.91 & 24.69 & -7.15 & 35.61 \\
G2320HDBL* & 222.9 & 0.179 & 39.90 & 23.65 & -4.67 & 31.79 \\
LG W2241S & 223.88 & 0.289 & 64.70 & 40.28 & -9.40 & 49.75 \\
ACER X163W & 225.76 & 0.117 & 26.41 & 14.80 & -3.37 & 21.62 \\
ACER V193HQ & 225.51 & 0.144 & 32.47 & 18.20 & -1.09 & 26.87 \\
PHILIPS 192E & 226.45 & 0.145 & 32.84 & 17.10 & -5.02 & 27.57 \\
SAMSUNG 943N & 226.11 & 0.247 & 55.85 & 32.75 & -1.24 & 45.22 \\
\hline
\end{tabular}

*BENQ

The first load is linear. Accordingly, the obtained currents track the voltage waveform. All other loads are nonlinear. Consequently they draw current with new harmonic components. Their amounts for all aforementioned nonlinear loads are measured and published in $[3,15]$. Therefore we used the data considering them as benchmarks known to power electronic community.

Table 1 summarizes the simulation results. All quantities in Table 1 are denoted according to annotation in equations (5)-(13). Two additional quantities are are total harmonic distortion of voltage $T H D_{\mathrm{V}}$, and current $T H D_{\mathrm{I}}$. They stand for total harmonic distortion of voltage and current waveforms, respectively. As specified in the previous section, $D_{\text {IEEE }}$ and $D_{1}$ are obtained when $Q_{\mathrm{B}}$ is replaced with $Q_{\text {IEEE }}$ and $Q_{1}$ in (15), respectively. For sake of completeness, Tab. 1 includes one more form for distortion power estimation. It relies on $T H D_{\mathrm{I}}$ and consequently is denoted as $D_{\mathrm{I}}=U_{1} \times T H D_{1}$, where $U_{1}$ denotes fundamental apparent power, according to [7].

The obtained data show three very important facts. Firstly, for all nonlinear loads $D_{\mathrm{B}}, D_{\text {IEEE }}$ and $D_{1}$ are greater than 0 . This confirms the postulate of the method. Secondly, all three definitions of $D\left(D_{\mathrm{B}}, D_{\text {IEEE }}\right.$ and $\left.D_{1}\right)$ applied at real loads give very similar results. Finally, it is obvious that the amount of $D$ should not be neglected. It has the same order of magnitude as active power in all energy saving devices. Overlooking $D$ what is the case in all utilities, causes considerable losses at power grid. After confirmation of the theory by simulation we are ready for practical application. The method was implemented using an off-the-shelf power meter. The subsequent section presents the results obtained on true loads.

\subsection{Measurement Results}

Applicability of the new method depends on possibility for implementation on a real commercial power meter. The only requirement was that the meter is capable to register $U, P$ and $Q_{\mathrm{B}}$. We used a meter produced by EWG electronics [16]. It is based on standard integrated circuit 71M6533 provided by MAXIM. The power meter completely fulfils IEC 62052-11 standard [17]. It offers data for $P, Q_{\mathrm{B}}$, and $U$ according to expressions (3), (5), and (6), respectively. However to calculate $D$ we need additional computation engine. The most common way was use a personal computer. However an additional effort was to interconnect it with the meter and to transfer measured data to PC for further acquisition.

Figure 2 illustrates the implemented set-up. The simplicity of the set-up is obvious. It consists of a meter, the load and PC. The measured data are read by standard optical head and transferred to PC through RS232 port. Original dedicated software managed data collection and transfer to Matlab script. Finally it calculates the distortion power.

Measurement results obtained for different type of energy saving lamps and PC monitors are reviewed in Tab.2. These loads are chosen due to their small nominal power (less then $100 \mathrm{~W}$ ). Moreover, energy saving lamps represent increasingly used nonlinear loads. At the moment, power consumed at different type of lamps reach up to $20 \%$ of the overall power consumption [18]. The obtained results absolutely show that the value of distortion power is sufficient to indicate if a consumer produces harmonic pollution or not. These results are in consistence with data recently published in [19] for similar types of energy saving lamps. Furthermore, the quantity of the pollution relates to the amount of registered distortion power. The main benefit of the method lays in its compatibility with the standard electronic power meters.

Practically, as a replacement for personal computer, an updated firmware could force the existing microprocessor embedded within the power meter to calculate D. Besides, the proposed solution can be implemented as a hardware upgrade of DSP that is a part of solid state power meters. We intend to enhance the features of the DSP block in our own ASIC power meter circuit that was designed in LEDA laboratory, University of Nis [20].

\section{CONCLUSION}

This paper proposed a new method capable to detect and locate nonlinear loads on a power grid. It is based on 
measurements of distortion power. The authors proved that existing diversities in definitions of distortion power are not obstacle to such approach. They compared applicability of four different definitions on seven practical examples. The simulation results demonstrated that differences between them do not compromise ability to locate a nonlinear load. In a simulated power system supplied with distorted all definitions resulted with an offset distortion power on linear loads. In order to preserve equipment from damage the utility has to keep voltage distortion under the tight limit defined by standard. Distorted voltage produces some small amount of distorted power on linear loads. That offset could be predicted for every type of distortion power definition. The method is established on using Budeanu's relationship between apparent, active, reactive, and distortion power. The method was prototyped using standard electronic power meter and personal computer. Then it was verified on set of nonlinear loads based on different energy saving lamps and PC monitors that commonly appears in offices and households.

This paper proves that power meters enhanced with possibility to register distortion power are sufficient and reliable indicator of customers with nonlinear loads. Applying the method the utility gets insight into location and the amount of pollution with harmonics entered to the grid at user's connection point. Accordingly, the utility gets a mechanism to extra-charge the harmonics producers. Large nonlinear loads are reality on grid and their devastating effects to the system should be prevented. The first step in that direction is their reliable location.

\section{Acknowledgment}

This work has been partly funded by the Serbian Ministry of Education and Science under the contract No. TR32004.

\section{REFERENCES}

[1] SINGH, G. K. : Power System Harmonics Research: a Survey, European Transactions on Electrical Power 19 (Aug 2007), $151-172$.

[2] ALHAZMI, Y.: Allocating Power Quality Monitors in Electrical Distribution Systems to Measure and Detect Harmonics Pollution, $\mathrm{PhD}$ dissertations, University of Waterloo, Ontario, Canada, 2010.

[3] WAKILEH, J. G. : Power Systems Harmonics, Springer, 2001.

[4] LEE, S.-PARK, J. W. : New Power Quality Index in a Distribution Power System by Using RMP Model, IEEE Tran. on Industrial Applications 46, No. 3 (May/June 2010), 1204-1211.

[5] WEBSTER, J. G.: The Measurement, Instrumentation, and Sensors Handbook, IEEE Press, 1999.

[6] CZARNECKI, L. S.: What is Wrong with Budeanu's Concept of Reactive and Distortion Power and why it Should be Abandoned, IEEE Transactions on Instrumentation and Measurement IM-36 No. 3 (Sep 1987).

[7] IEEE Standard Definitions for the Measurement of Electric Power Quantities Under Sinusoidal, Nonsinusoidal, Balanced, Or Unbalanced Conditions, IEEE Std 1459-2010, March 2010.
[8] HARTMAN, M. : Orthogonality of Functions Describing Power States in Electrical Circuits in Budeanu's Concept, Proc. of the International School on Nonsinusoidal Current and Compensation, Lagow, Poland, June 15-18, 2010.

[9] TAGARE, D. M.: Reactive Power Management, McGraw-Hill Education, 2007.

10] EMANUEL, A. E. : Summary of IEEE Standard 1459: Definitions for the Measurement of Electric Power Quantities under Sinusoidal, Nonsinusoidal, Balanced, or Unbalanced Conditions, IEEE Tran. On Industrial Applications 40 No. 3 (May 2004), 869-876.

[11] XU, W.-LIU, X.-LIU, Y.: An Investigation on the Validity of Power-Direction Method for Harmonic Source Determination, IEEE Trans. Power Delivery 18 No. 1 (Jan 2003), 214-219.

[12] CHUN, L.-XU, W.-TAYJASANANT, T.: A Critical Impedance Based Method for Identifying Harmonic Sources, IEEE Trans. Power Del. 19 No. 2 (Apr 2004), 671-678.

[13] BARBARO, P. V.-CATAliotTi, A.-COSENTINO, V.NUCCIO, S.: A New Technique to Detect Harmonic Sources in Polluted Power Systems, Proc. of the XVIII IMEKO World Congress, Rio de JaneiroBrazil, 2006.

14] CAtaliotti, A.-Cosentino, V.: A Single-Point Approach based on IEEE 1459-2000 for the Identification of Detection of Prevailing Harmonic Sources in Distorted Three Phase Power Systems, Proc. of the Metrology and Measurement Systems, 2009.

15] WEI, Z. : Compact Fluorescent Lamps Phase Dependency Modelling and Harmonic Assessment of their Widespread Use in Distribution Systems, PhD dissertations, University of Canterbury, Christchurch, New Zealand, 2009.

[16] EWG - Multi Metering Solutions, www.ewg.rs.

[17] Electricity Metering Equipment (AC) - General Requirements, Tests and Test Conditions - Part 11: Metering equipment, IEC 62052-11, Feb 2003.

[18] HAMMERSCHMIDT, C. : Research Project Illuminates OLEDs' Industrial Future, EETimes (Dec 2011).

[19] DimitriJeviĆ, M.-LiTOVsKI, V.: Power Factor and Distortion Measuring for Small Loads Using USB Acquisition Module, Journal of Circuits Systems and Computers 20 No. No 5 (Aug 2011), 867-880, World Scientific Publishing Co. Pte. Ltd., Singapore.

[20] http://leda.elfak.ni.ac.rs/projects/IMPEG/impeg.htm.

Received 10 January 2013

Predrag Petković was born in Čačak, Serbia. He received $\mathrm{PhD}$ degree from the University of Niš, in 1990. He is currently full professor at University of Niš, Faculty of Electronic Engineering. His research interests include ASIC design, optimisation, modeling, and simulation focusing on symbolic circuit simulation, power quality and mixed signal integrated circuits design. Prof. Petković is member of IEEE, Yugoslav Simulation Society. He is the author of about 200 publications. He received several awards for his scientific and educational work.

Dejan Stevanović was born in Vranje, Serbia, in 1982. He received the Dipl-Ing (MS) degree from the University of Niš, in 2007. He is currently Research assistant at Innovation Centre of Advanced Technologies CNT (ICNT). Mr. Stevanovi has been member of Leda laboratory since 2007. His current research interests are power quality and digital integrated circuits design. He is the author of about 20 publications. She received several rewards for her scientific research. 